\title{
Análise Bibliométrica e de Rede dos 40 Anos da Revista Psicologia: Ciência e Profissão
}

\author{
Rodrigo de Oliveira Machado ${ }^{1}$ \\ ${ }^{1}$ Universidade Federal de Sergipe, SE, Brasil. \\ Jingyuan $\mathrm{Yu}^{2}$ \\ ${ }^{2}$ Univesitat Autònoma de Barcelona, BCN, Espanha.
}

\author{
Miquel Domènech ${ }^{2}$ \\ ${ }^{2}$ Univesitat Autònoma de Barcelona, BCN, Espanha. \\ Juan Muñoz-Justícia ${ }^{2}$ \\ ${ }^{2}$ Univesitat Autònoma de Barcelona, BCN, Espanha.
}

Resumo: No ano de celebração dos 40 anos da revista Psicologia: Ciência e Profissão cabe verificar como esta revista foi delineando a sua própria história e do campo da Psicologia no Brasil. Neste sentido, o presente artigo teve como objetivo delimitar os principais autores, instituições e palavras-chave que trouxeram a revista até o momento atual. Partindo de uma análise bibliométrica e de redes, obteve-se como resultado os temas, autores e instituições que tiveram protagonismo e a interação entre esses atores. As conclusões apontam que a revista assume uma postura epistemológica pluralista e que tanto os aspectos científicos como os relacionados a práticas e atuação dos profissionais se mantêm ao longo desses 40 anos.

Palavras-chave: História da Psicologia, Análise Bibliométrica, Análise de Redes.

\section{Bibliometric and Network Analysis of the 40 Years of the Journal Psicologia: Ciência e Profissão}

\begin{abstract}
In the year of celebration of the $40^{\text {th }}$ anniversary of the journal Psicologia: Ciência $e$ Profissão (Psychology: Science and Profession), it is necessary to review how this journal has been outlining its own history and the field of Psychology in Brazil. In this sense, this article aimed to delimit which were the main authors, institutions and keywords that brought the journal to the present moment. Based on a bibliometric and network analysis, it was obtained as a result which themes, authors and institutions had protagonism and interaction among these actors. The conclusions indicate that the journal assumes a pluralist epistemological posture and that both the scientific aspects and those related to the practices and performance of professionals are maintained throughout these 40 years.
\end{abstract}

Keywords: History of Psychology, Bibliometric Analysis, Network Analysis. 


\title{
Análisis Bibliométrico y de Redes de los 40 Años de la Revista Psicologia: Ciência e Profissão
}

\begin{abstract}
Resumen: En el año en que se celebra el $40^{\circ}$ aniversario de la revista Psicologia: Ciência e Profissão (Psicología: Ciencia y Profesión), es necesario revisar cómo esta revista ha ido perfilando su propia historia y el campo de la Psicología en el Brasil. En este sentido, este artículo pretendía delimitar cuáles fueron los principales autores, instituciones y palabras clave que llevaron a la revista al momento actual. Basándose en un análisis bibliométrico y de redes, se obtuvo como resultado qué temas, autores e instituciones eran los principales e interacción entre estos actores. Las conclusiones indican que la revista asume una postura epistemológica pluralista y que tanto los aspectos científicos como los relacionados con las prácticas y el desempeño de los profesionales se mantienen a lo largo de estos 40 años.
\end{abstract}

Palabras-clave: Historia de la Psicología, Análisis Bibliométrico, Análisis de Red.

\section{Introdução}

O início da história da Psicologia como disciplina independente dos ramos da Filosofia e da Medicina remonta ao ano de 1879 , tendo como ato representativo a criação do primeiro espaço destinado a pesquisa neste novo campo que emergia. Os experimentos realizados no Laboratório de Leipizg, que tinha Whilhem Wundt como expoente, considerado por muitos como pai da Psicologia, forneceram a distinção necessária para que esse evento entrasse nos cânones da ciência moderna. Este primeiro passo da formação do campo da Psicologia é um recorte histórico aprendido comumente em qualquer classe de História da Psicologia, e evidentemente, como qualquer historiografia, os recortes e modos de interpretação de eventos sempre serão parciais e enviesados pelos objetivos daqueles que os escrevem. O apagamento histórico da Völkerpsychologie (psicologia dos povos) do próprio Wundt serve como exemplo de como escrever a história de determinados eventos ou períodos considerados importantes é sempre carregado por uma complexidade que invariavelmente assume alguns indicadores e deixa à margem outros (Farr, 2013).

Embora o presente artigo não se preste a fazer um acompanhamento histórico clássico, o exemplo da inauguração da Psicologia serve como figura para apresentar os elementos que sempre estão orientando a construção de uma narrativa histórica, ou seja, a não neutralidade e a parcialidade dos dados que a sustentam. Esse caráter contingencial da análise histórica remete a uma inconclusibilidade sobre o tema e traz sentido para avaliações sequenciais daquilo que denomina determinados períodos e, ao longo do tempo, podem tomar a forma substantiva de verdade. Os limites epistemológicos estão presentes em qualquer trabalho que tenha por objetivo descrever a história de um evento, instituição, pessoas ou qualquer outra entidade. A história enquanto narrativa, ainda que importante para compreender um todo complexo, pode nos oferecer visões demasiado parciais.

Outros formatos para delimitar e expressar a construção histórica auxiliam na compreensão de como uma área do conhecimento se desenvolve, sendo alguns amparados em marcadores mais objetivos e quantificáveis. Neste sentido, a bibliometria se consagra como disciplina que, por meio de captação, organização e análise de dados, consegue fornecer retratos instantâneos de períodos históricos determinados. A diversidade e a quantidade de artigos científicos que estão disponíveis desde a proliferação das tecnologias de informação e comunicação (TIC) transformaram não somente a forma de divulgação do conhecimento científico, mas também as maneiras de compreender a história desse conhecimento, e a bibliometria surge como resposta para tal desafio.

No esteio dessas considerações, o período de comemoração dos 40 anos da revista Psicologia: Ciência e Profissão (PCP) apresenta-se como oportunidade para revisitar a trajetória da própria revista e de como o campo da Psicologia vem sendo representado no Brasil. A importância deste periódico se verifica não somente pela qualidade do material publicado 
ao longo dos anos, ou de avaliações externas, como o Qualis A1, mas sobretudo pelo fato de estar diretamente vinculada ao Conselho Federal de Psicologia, isto é, a principal organização da classe profissional no país. Em outras palavras, analisar os dados referentes à PCP possibilita, além de compreender o desenvolvimento de publicações no campo da Psicologia, entender os caminhos que a profissão, e os estudos a ela pertencentes, foram tomando ao longo deste quase meio século de história.

Estabelecida como objetivo central a representação de um panorama complexo dessa trajetória, é necessário ressaltar algumas escolhas metodológicas que foram substanciais para a efetivação do presente trabalho, sendo a principal delas postular o estudo dentro do campo das análises bibliométricas e cienciométricas. As variáveis assumidas para a realização dessa investigação, assim como os motivos para suas escolhas, estão descritas com maior profundidade no apartado metodológico. Em termos gerais, o leitor encontrará no decorrer do texto aqueles que foram os principais autores, instituições e palavras-chaves que constituem as publicações da revista.

\section{Antecedentes históricos da Psicologia no Brasil e o surgimento das revistas científicas}

Conforme citado anteriormente, o nascimento da Psicologia como área distinta da ciência moderna já estava marcado pela tensão entre diferentes visões do que se tratava essa disciplina, os objetivos que a orientavam e as limitações próprias que se encontraria (Farr, 2013). A formação das diferentes escolas psicológicas no decorrer dos anos foi tentando estabilizar um paradigma comum, porém a contestação de uma visão epistemológica única ao campo não tomou forma de realidade, e, devido às idiossincrasias pertinentes ao objeto elementar de estudo, provavelmente nunca o logrará. Em outras palavras, a existência da Psicologia como área de estudos que vislumbra compreender o ser humano, seja da perspectiva das relações sociais, emoções, cognição ou qualquer outro tema que costuma preocupar os acadêmicos, estará sempre marcada pela incompletude de análises de caráter fisiológico ou da ordem filosófica/sociológica.

Um resgate histórico das tentativas elaboradas a partir das escolas psicológicas pode ser consultado em qualquer manual geral do campo. As implicações e correspondências dessas vertentes da Psicologia no contexto brasileiro demonstra a força dessas correntes em diversos momentos da produção psicológica nacional. A psicofisiologia de Wundt e Pavlov serviu como modelo para os escritos reconhecidos como do campo da Psicologia durante o período de 1900 a 1920, que tinha sobretudo médicos como autores (Soares, 2010).

O período posterior à década de 1920 indica a utilização do saber psicológico como eixo a ser considerado com maior atenção no que diz respeito a práticas educacionais (Jacó-Vilela, 2012; Soares, 2010). O behaviorismo norte-americano e sua ênfase na mudança comportamental como fundador de uma nova sociedade também apareciam vinculados a projetos de educação (Skinner, 1972). Embora não se possa comparar a participação da Psicologia na educação brasileira com o projeto comportamentalista planejado nos Estados Unidos, nota-se uma correspondência entre valores e a finalidade do saber psicológico para o campo da Pedagogia.

O modelo da Psicologia norte-americana também teve influência no desenvolvimento da área organizacional e de testes psicológicos no Brasil, sendo a industrialização brasileira tardia o evento propulsor para a formação dessas áreas e sua expansão para além do âmbito acadêmico (Zanelli, 1995). Em 1925, foi fundado o Instituto de Seleção e Orientação Profissional de Pernambuco, com o intuito de vincular as necessidades do mundo do trabalho às ferramentas e teorias oriundas da Psicologia.

Em resumo, os períodos de 1900-1920 e 1920-1960 podem também ser divididos de acordo com os principais autores do campo nesses anos. O primeiro período tinha médicos como protagonistas, os quais eram responsáveis pela publicação de ensaios, teses e laboratórios que corroboravam a psicologia científica da época. No segundo período, os autores eram sobretudo educadores que se aproximavam da ciência psicológica para fundamentar os trabalhos realizados junto a escolas normais, tendo destaque a orientação profissional e os testes desenvolvidos para este enfoque (Soares, 2010).

A divulgação científica em formato de revistas ocorre de maneira esparsa durante a primeira metade do século XX. Em 1928, a Revista Brasileira de Psicanálise foi o primeiro periódico do campo da Psicologia a ser lançado no país, sendo seguido pelo Boletim de Psicologia, em 1948, e a revista Arquivos Brasileiros de Psicotécnica, em 1949. 
Quanto aos conteúdos discutidos no período, existem poucos estudos que revisem esse material, contudo, entre 1930 e 1962, a produção acadêmica encontra-se numa totalidade de 405 produtos, que se subdividem em quatro grandes categorias: obras/ publicações, ensino, aplicação e estudos/pesquisas. Estas categorias foram compostas por uma série de materiais que tiveram como característica ser publicados sistematicamente, ou seja, não se referem somente a artigos ou livros publicados de maneira esporádica. A concentração dessa produção se encontra sobretudo nas capitais do país, Rio de Janeiro e Distrito Federal, e em São Paulo (Antunes, 2006). Entre as instituições responsáveis pelas obras, constata-se uma maioria de natureza pública (Antunes, 2006).

No decorrer dos anos 1940, começaram a surgir os primeiros periódicos brasileiros dedicados à Psicologia, seguidos, na década seguinte, da inauguração dos cursos de Psicologia em São Paulo e Rio Grande do Sul (Feres-Carneiro, 2011; Scarparo, 2011). A celebração desses dois momentos, em conjunto com as associações de psicólogos que já existiam, colaborou para que o caminho profissional da Psicologia fosse regularizado a partir de 1962.

As organizações dos psicólogos em torno de associações e sociedades, em conjunto com as modificações políticas e sociais decorrentes daquele momento histórico, possibilitaram a criação dos Conselhos Regionais e Federal de Psicologia (Baptista, 2010). A lei de criação dos conselhos é de 1971, e a primeira eleição dos conselheiros, para a gestão 1973/1975, ocorreu dois anos depois, em 20 de dezembro de 1973, sendo este o ano de criação do Conselho Federal de Psicologia.

A PCP teve seu primeiro número publicado em 1979, e adotou regularidade em sua publicação a partir de 1981 (Gomes \& Gauer, 2010). Devido a sua origem, a revista se notabiliza pelo caráter institucional, publicando materiais que se conectam com a vertente profissional e científica da Psicologia. Conforme ressaltado por Campos \& Bernardes (2005), a periodicidade da revista esteve comprometida até meados da década de 1990, sendo esta questão resolvida a partir de 1997. Em parte, a regularidade da revista se resolve a partir das mudanças promovidas pela Capes, que estipula critérios (Qualis) para avaliar as revistas científicas (Guedes, 2010).
Em 2005, no aniversário de 25 anos da revista, foi proposta uma recapitulação histórica, que a dividiu em quatro fases e que fornece indicadores que correspondem à linha editorial institucional e científica em que a PCP transita. As linhas editoriais que foram categorizadas até aquele momento se definiam pela ênfase em determinados temas: $1^{\text {a }}$ fase, de 1979 a 1983 - história da Psicologia e pesquisas da área realizadas no país; 2a fase, de 1984 a 1991 - relevância social do trabalho realizado pelos psicólogos e discussão de questões da categoria; 3 a fase, de 1992 a 1996 - divulgação de artigos com ênfase em temas socialmente importantes e trabalhos científicos; $4^{\text {a }}$ fase, de 1997 a 2004 - publicação de artigos considerados de primeira linha, com o objetivo de consolidar a revista entre as melhores da área, contudo mantendo temas socialmente relevantes (Campos \& Bernardes, 2005). Por sua vez, na comemoração dos 30 anos da revista, foi realizada uma análise bibliográfica do período de 2005 a 2009, que demonstrou a permanência de uma periodicidade e o aumento do número de artigos publicados (Sampaio, Serradas, \& Santos, 2010).

\section{Método}

Entre as formas de conhecer determinada área do conhecimento, a análise bibliométrica é especialmente adequada para identificar os contornos que caracterizam o campo em investigação. Estudos bibliométricos e cienciométricos têm dado contribuição significativa para a compreensão de algumas das dinâmicas que atuam no campo da ciência, caracterizando os processos pelos quais a construção do conhecimento é desenvolvida e funciona (Callon, Law, \& Rip, 1986).

Especificamente, os avanços na cientometria contribuíram para a compreensão e a clarificação de diferentes aspectos relacionados à prática científica, como o aparecimento e o desenvolvimento de revoluções científicas (De Langhe, 2017) ou a delimitação de campos de pesquisa dentro de determinada área - ver os casos de segurança da informação (Lee, 2008), nanotecnologia (Zhou \& Leydesdorff, 2006) ou informática (Uriona-Maldonado, dos Santos, \& Varvakis, 2012). Em termos gerais, a cientometria e, sobretudo, os softwares desenvolvidos dentro da área contribuem para a coleta e a análise de grandes quantidades de dados referentes a publicações científicas, a fim de torná-los visíveis e identificáveis. 


\section{Dados}

Os dados foram coletados através do pacote rscielo (Meireles, Silva, \& Barbosa, 2019), do programa $R$ ( $R$ Core Team, 2019), em 30 de outubro de 2019, na base de dados SciELO, utilizando as publicações da revista Psicologia: Ciência e Profissão como critério de busca. Foi recuperado um total de 1.727 registros, cobrindo os anos de 1982 a 2019. Em seguida, realizou-se uma primeira limpeza de dados, mantendo apenas as referências do tipo "artigo", reduzindo-se a um total de 1.482 registros. A segunda limpeza consistiu na homogeneização de nomes de autores (as várias formas como os nomes poderiam aparecer), universidades e palavras-chave.

\section{Análise descritiva}

A análise foi realizada com diferentes funções do programa $R$. Foi feita uma análise das frequências de autor, ou seja, os autores com maior número de textos, palavras-chave e universidades (dependendo da filiação institucional dos signatários dos artigos). A análise foi feita para dados totais e por períodos de nove anos, não incluindo os dois primeiros anos da revista (1982 e 1983) devido ao pequeno número de artigos que apareceram nesses anos iniciais (4 e 8, respectivamente). Os períodos resultantes foram 1984-1992, 1993-2001, 2002-2010 e 2011-2019.

\section{Análise colaborativa}

Para mostrar a rede de colaboração presente nas publicações da revista, foi realizada análise da colaboração, baseada na coautoria dos artigos, com o pacote $R$ Bibliometrix (Aria e Cuccurullo, 2017). Foram realizadas duas análises, utilizando no primeiro caso os autores dos artigos como unidade de análise, obtendo-se assim a matriz de colaboração entre os autores; e no segundo caso utilizando as instituições de filiação dos autores como unidade de análise, obtendo-se assim a matriz de colaboração entre as universidades. Os resultados de ambas as matrizes foram exportados para representação gráfica com o programa Gephi (Bastian, Heymann, \& Jacomy, 2009), selecionando os 50 autores e as 30 universidades com o maior número de colaborações.

\section{Análise temática}

Para caracterizar a estrutura temática da revista, foi realizada uma análise de co-ocorrência de palavras-chave com o pacote $R$ Bibliometrix (Aria \& Cuccurullo, 2017). Como as palavras-chave são uma forma de caracterização do artigo, sua co-ocorrência fornece uma visão sobre os tópicos cobertos em um conjunto de publicações e as relações entre elas (de la Hoz-Correa, Muñoz-Leiva, \& Bakucz, 2018).

\section{Resultados}

A apresentação do conjunto de resultados que emergiram da busca realizada na PCP seguirá a ordem de primeiramente mostrar como, ao longo dos 40 anos da revista, autores, instituições e temas foram marcando presença nas publicações do periódico em distintos momentos dessa trajetória. Conforme explicitado anteriormente, essa divisão temporal ocorreu em intervalos de oito anos. Em seguida, os dados apresentados demonstrarão os principais expoentes em cada uma dessas três categorias (autoria, instituição e palavras-chave), configurando assim as referências no que diz respeito a este quase meio século da revista. Por fim, novamente tais categorias estão representadas através de redes que revelam a proximidade entre elas.

Tabela 1.

Artigos, autores, universidades e palavras-chave por período.

\begin{tabular}{ccccc}
\hline Período & Artigos & Autores & Instituições & Keywords \\
\hline $1984-1992$ & 141 & 139 & 70 & $0^{1}$ \\
$1993-2001$ & 166 & 190 & 90 & 142 \\
$2002-2010$ & 469 & 845 & 205 & 1.088 \\
$2011-2019$ & 693 & 1.399 & 258 & 1.258 \\
\hline
\end{tabular}

\footnotetext{
${ }^{1}$ O primeiro ano sobre o qual temos informações de palavras-chave é 2000 (volume 3).
} 
Tabela 2.

Produção das instituições por intervalo temporal de oito anos.

\begin{tabular}{|c|c|}
\hline Instituição & $\mathrm{N}$ \\
\hline \multicolumn{2}{|l|}{ 1984-1992 } \\
\hline Universidade de São Paulo & 25 \\
\hline Universidade de Brasília & 10 \\
\hline Universidade Federal da Paraíba & 7 \\
\hline Pontifícia Universidade Católica de São Paulo & 6 \\
\hline Universidade Estadual de Campinas & 6 \\
\hline Universidade Federal de Minas Gerais & 5 \\
\hline Universidade Federal de Pernambuco & 5 \\
\hline $\begin{array}{l}\text { Pontifícia Universidade Católica do } \\
\text { Rio Grande do Sul }\end{array}$ & 4 \\
\hline Crp-06 & 3 \\
\hline $\begin{array}{l}\text { Pontifícia Universidade Católica do } \\
\text { Rio de Janeiro }\end{array}$ & 3 \\
\hline Universidade Federal Fluminense & 3 \\
\hline Diesat & 2 \\
\hline $\begin{array}{l}\text { Secretaria Municipal de Educação do } \\
\text { Rio de Janeiro }\end{array}$ & 2 \\
\hline $\begin{array}{l}\text { Sindicato dos Psicólogos no Estado de } \\
\text { São Paulo }\end{array}$ & 2 \\
\hline Universidade Católica de Goiás & 2 \\
\hline Universidade de Mogi das Cruzes & 2 \\
\hline Universidade Estadual de Maringá & 2 \\
\hline Universidade Federal de Santa Catarina & 2 \\
\hline Universidade Federal do Rio de Janeiro & 2 \\
\hline Universidade Federal do Rio Grande do Sul & 2 \\
\hline \multicolumn{2}{|l|}{$1993-2001$} \\
\hline Universidade de São Paulo & 15 \\
\hline Universidade Federal de Minas Gerais & 12 \\
\hline Pontifícia Universidade Católica de São Paulo & 10 \\
\hline Universidade de Brasília & 10 \\
\hline Universidade Federal de Pernambuco & 6 \\
\hline Fundação Oswaldo Cruz & 5 \\
\hline Universidade Federal de São Carlos & 5 \\
\hline Universidade Federal do Pará & 5 \\
\hline Universidade Federal do Rio Grande do Sul & 5 \\
\hline Universidade Federal do Rio de Janeiro & 4 \\
\hline Universidade Estadual Paulista J. M. Filho & 3 \\
\hline Universidade Federal da Bahia & 3 \\
\hline Universidade Federal do Paraná & 3 \\
\hline Universidade Regional de Blumenau - Furb & 3 \\
\hline
\end{tabular}

continua... ...continuação

\begin{tabular}{lc}
\hline Instituição & $\mathrm{N}$ \\
\hline Detran & 2
\end{tabular}

Pontifícia Universidade Católica de Campinas

Pontifícia Universidade Católica do

Rio de Janeiro

2

Universidade Católica de Brasília

Universidade do Estado do Rio de Janeiro 2

\begin{tabular}{ll}
\hline \multicolumn{2}{c}{$2002-2010$} \\
\hline Universidade de São Paulo
\end{tabular}

Universidade Federal do Rio Grande do Sul $\quad 31$

Universidade Federal da Paraíba 21

Universidade Federal de Santa Catarina $\quad 20$

Universidade São Francisco 19

Pontifícia Universidade Católica do 18

Rio de Janeiro

Universidade de Brasília $\quad 16$

Universidade Federal da Bahia $\quad 16$

Universidade Federal de Pernambuco 14

Pontifícia Universidade Católica de 12

Minas Gerais

Universidade do Estado do Rio de Janeiro 12

Universidade Estadual Paulista J. M. Filho 12

Universidade Federal de Minas Gerais $\quad 11$

Pontifícia Universidade Católica de São Paulo 10

Pontifícia Universidade Católica do

Rio Grande do Sul

Universidade Estadual da Paraíba $\quad 10$

Universidade Federal do Espírito Santo 10

Universidade Federal do Rio Grande do Norte $\quad 10$

Universidade Federal do Ceará 8

Universidade Federal do Pará 8

\begin{tabular}{ll}
\hline \multicolumn{2}{c}{ 2011-2019 } \\
\hline Universidade de São Paulo & 90 \\
Universidade Federal do Rio Grande do Sul & 89 \\
Universidade Federal de Santa Catarina & 77 \\
Universidade Federal da Paraíba & 62 \\
Universidade Federal de Santa Maria & 52 \\
Universidade de Brasília & 51 \\
Universidade Estadual Paulista J. M. Filho & 40 \\
Universidade Federal do Rio Grande do Norte & 37 \\
Universidade Federal do Rio de Janeiro & 32 \\
\hline
\end{tabular}

continua... 


\begin{tabular}{ll}
...continuação & $\mathrm{N}$ \\
\hline Instituição & 28 \\
\hline Universidade Federal de Uberlândia & 27 \\
Universidade do Estado do Rio de Janeiro & 27 \\
Universidade Federal do Ceará & 25 \\
Pontifícia Universidade Católica do & \\
Rio Grande do Sul & 24 \\
Pontifícia Universidade Católica de & \\
Campinas & 24 \\
Universidade do Vale do Rio dos Sinos & 24 \\
Universidade Federal do Espírito Santo & 22 \\
Universidade Federal Fluminense & 21 \\
Universidade Federal de São Paulo & 19 \\
Universidade Federal do Paraná & 18 \\
Universidade São Francisco & \\
\hline
\end{tabular}

Tabela 3.

Autores com o maior número de textos por período.

\begin{tabular}{ll}
\hline Autor & $\mathrm{n}$ \\
\hline \multicolumn{1}{c}{$1984-1992$} & \\
\hline Marlene Guirado & 7 \\
Antônio Roazzi & 4 \\
Carlos Roberto Drawin & 4 \\
Jairo E. Borges Andrade & 4 \\
Sérgio Antônio da Silva Leite & 4 \\
Almir Del Prette & 3 \\
Antônio Virgilio Bittencourt Bastos & 3 \\
Eunice M. L. Soriano de Alencar & 3 \\
Cecília Maria B. Coimbra & 2 \\
José Sollero Neto & 2 \\
Juarez Alves & 2 \\
Lorismário E. Simonassi & 2 \\
Maria Helena Souza Patto & 2 \\
Marisa Faermann Elsirik & 2 \\
Marta Cristina Ortiz & 2 \\
Paula Inez Cunha Gomide & 2 \\
Sylvia Leser de Mello & 2 \\
Thereza Pontual de Lemos Mettel & 2 \\
\hline
\end{tabular}

continua... ...continuação

\begin{tabular}{|c|c|}
\hline Autor & $\mathrm{n}$ \\
\hline \multicolumn{2}{|l|}{$1993-2001$} \\
\hline Aderson Luiz Costa Jr. & 4 \\
\hline Elaine Pedreira Rabinovich & 3 \\
\hline Alexandre Dittrich & 2 \\
\hline Ana Maria Almeida Carvalho & 2 \\
\hline André Maurício Monteiro & 2 \\
\hline Carmem Lígia Lochins Grisci & 2 \\
\hline Enrique Carbonell & 2 \\
\hline Laura dos Santos Gomes & 2 \\
\hline Luciana Fim Wickert & 2 \\
\hline Luis Montoro & 2 \\
\hline Maria Helena Hoffmann & 2 \\
\hline Maria Joana Mäder & 2 \\
\hline Núbia Schaper Santos & 2 \\
\hline Patty Fidelis de Almeida & 2 \\
\hline Paulo Wenderson Teixeira Moraes & 2 \\
\hline Rafael Andrés Villari & 2 \\
\hline Roberto Henrique Amorim de Medeiros & 2 \\
\hline Tatiana Ramminger & 2 \\
\hline \multicolumn{2}{|l|}{$2002-2010$} \\
\hline Ana Maria Nicolaci-Da-Costa & 8 \\
\hline Ana Paula Porto Noronha & 7 \\
\hline Maria da Penha De Lima Coutinho & 7 \\
\hline Denise D’Áurea-Tardeli & 5 \\
\hline Daniel Bartholomeu & 3 \\
\hline Dulce Helena Penna Soares & 3 \\
\hline Fabián Javier Marín Rueda & 3 \\
\hline Fermino Fernandes Sisto & 3 \\
\hline Fernando Luis González Rey & 3 \\
\hline Magda Dimenstein & 3 \\
\hline Manoel Antônio dos Santos & 3 \\
\hline Sérgio Gomes da Silva & 3 \\
\hline Valdiney Veloso Gouveia & 3 \\
\hline Aderson Luiz Costa Jr. & 2 \\
\hline Antônio Virgilio Bittencourt Bastos & 2 \\
\hline
\end{tabular}




\begin{tabular}{lc}
...continuação & $\mathrm{n}$ \\
\hline Autor & \\
\hline \multicolumn{1}{c}{ 2011-2019 } \\
\hline Neuza Guareschi & 11 \\
Roberto Moraes Cruz & 11 \\
Magda Dimenstein & 9 \\
Manoel Antônio dos Santos & 9 \\
Valdiney Veloso Gouveia & 9 \\
Dorian Mônica Arpini & 8 \\
Ana Cristina Garcia Dias & 7 \\
Pedro Paulo Gastalho de Bicalho & 6 \\
Ana Maria Lopez Calvo de Feijoo & 5 \\
Daniela Ribeiro Schneider & 5 \\
Maria de Fátima Pereira Alberto & 5 \\
Clarissa Tochetto de Oliveira & 4 \\
Fabíola Langaro & 4 \\
João Paulo Macedo & 4 \\
Leila Maria Torraca de Brito & 4 \\
Marivete Gesser & 4 \\
Raquel Souza Lobo Guzzo & 4 \\
Thiago Antônio Avellar de Aquino & 4 \\
\hline
\end{tabular}

Tabela 4.

Autores com o maior número de textos na revista (mais de 5 artigos).

\begin{tabular}{|c|c|c|c|}
\hline & & & \\
\hline Autor & $\begin{array}{l}\text { Número } \\
\text { de textos }\end{array}$ & $\begin{array}{l}\text { K10 Grande do Sul } \\
\text { Universidade do Estado do Rio de Janeiro }\end{array}$ & 43 \\
\hline Manoel Antônio dos Santos & 13 & Universidade Federal do Espírito Santo & 39 \\
\hline Neuza Guareschi & 13 & Pontifícia Universidade Católica de São Paulo & 38 \\
\hline Magda Dimenstein & 12 & Universidade Federal da Bahia & 37 \\
\hline Roberto Moraes Cruz & 12 & Universidade Federal do Ceará & 37 \\
\hline Valdiney Veloso Gouveia & 12 & Universidade São Francisco & 37 \\
\hline Ana Maria Nicolaci-Da-Costa & 10 & Universidade Federal de Pernambuco & 36 \\
\hline Ana Paula Porto Noronha & 10 & Universidade Federal de Uberlândia & 36 \\
\hline Dorian Mônica Arpini & 9 & Universidade Federal Fluminense & 33 \\
\hline Maria da Penha de Lima Coutinho & 9 & Universidade do Vale do Rio dos Sinos & 32 \\
\hline Ana Cristina Garcia Dias & 7 & $\begin{array}{l}\text { Pontifícia Universidade Católica do } \\
\text { Rio de Janeiro }\end{array}$ & 31 \\
\hline Antônio Virgilio Bittencourt Bastos & $\frac{7}{\text { continue }}$ & $\begin{array}{l}\text { Pontifícia Universidade Católica de } \\
\text { Campinas }\end{array}$ & 30 \\
\hline
\end{tabular}

\begin{tabular}{|c|c|}
\hline $\begin{array}{l}\text { Nú } \\
\text { de }\end{array}$ & $\begin{array}{l}\text { Júmero } \\
\text { e textos }\end{array}$ \\
\hline Maria de Fátima Pereira Alberto & 7 \\
\hline Marlene Guirado & 7 \\
\hline Aderson Luiz Costa Jr. & 6 \\
\hline Antônio Roazzi & 6 \\
\hline Jairo E. Borges Andrade & 6 \\
\hline Leila Maria Torraca de Brito & 6 \\
\hline Pedro Paulo Gastalho de Bicalho & 6 \\
\hline \multicolumn{2}{|l|}{$\begin{array}{l}\text { Tabela } 5 . \\
\text { Universidades com o maior número de aparições. }\end{array}$} \\
\hline Universidade & $\mathrm{n}$ \\
\hline Universidade de São Paulo & 189 \\
\hline Universidade Federal do Rio Grande do Sul & 131 \\
\hline Universidade Federal de Santa Catarina & 101 \\
\hline Universidade Federal da Paraíba & 93 \\
\hline Universidade de Brasilia & 90 \\
\hline Universidade Estadual Paulista J. M. Filho & 56 \\
\hline Universidade Federal de Santa Maria & 53 \\
\hline Universidade Federal de Minas Gerais & 50 \\
\hline Universidade Federal do Rio Grande do Norte & te 50 \\
\hline Universidade Federal do Rio de Janeiro & 47 \\
\hline $\begin{array}{l}\text { Pontifícia Universidade Católica do } \\
\text { Rio Grande do Sul }\end{array}$ & 43 \\
\hline Universidade do Estado do Rio de Janeiro & 43 \\
\hline Universidade Federal do Espírito Santo & 39 \\
\hline Pontifícia Universidade Católica de São Paulo & lo 38 \\
\hline Universidade Federal da Bahia & 37 \\
\hline Universidade Federal do Ceará & 37 \\
\hline Universidade São Francisco & 37 \\
\hline Universidade Federal de Pernambuco & 36 \\
\hline Universidade Federal de Uberlândia & 36 \\
\hline Universidade Federal Fluminense & 33 \\
\hline Universidade do Vale do Rio dos Sinos & 32 \\
\hline $\begin{array}{l}\text { Pontifícia Universidade Católica do } \\
\text { Rio de Janeiro }\end{array}$ & 31 \\
\hline $\begin{array}{l}\text { Pontifícia Universidade Católica de } \\
\text { Campinas }\end{array}$ & 30 \\
\hline
\end{tabular}


Tabela 6.

Palavras-chave mais frequentes (por período).

\begin{tabular}{|c|c|}
\hline Palavra-chave & $\mathrm{n}$ \\
\hline \multicolumn{2}{|c|}{ 1993-2001 } \\
\hline psicanálise & 6 \\
\hline clínica & 3 \\
\hline ética & 3 \\
\hline psicologia & 3 \\
\hline subjetividade & 3 \\
\hline autonomia & 2 \\
\hline criança & 2 \\
\hline inconsciente & 2 \\
\hline saúde mental & 2 \\
\hline \multicolumn{2}{|c|}{$2002-2010$} \\
\hline saúde mental & 31 \\
\hline psicologia & 25 \\
\hline subjetividade & 22 \\
\hline psicanálise & 19 \\
\hline reforma psiquiátrica & 17 \\
\hline avaliação psicológica & 15 \\
\hline representação social & 15 \\
\hline trabalho & 15 \\
\hline adolescência & 13 \\
\hline adolescente & 11 \\
\hline família & 10 \\
\hline psicólogo & 10 \\
\hline formação do psicólogo & 9 \\
\hline identidade & 9 \\
\hline psicologia social & 9 \\
\hline educação & 8 \\
\hline grupo & 8 \\
\hline psicoterapia & 8 \\
\hline moral & 7 \\
\hline saúde & 7 \\
\hline testes psicológicos & 7 \\
\hline violência & 7 \\
\hline \multicolumn{2}{|c|}{ 2011-2019 } \\
\hline psicologia & 70 \\
\hline psicanálise & 40 \\
\hline saúde mental & 31 \\
\hline avaliação psicológica & 27 \\
\hline atuação do psicólogo & 26 \\
\hline trabalho & 25 \\
\hline formação do psicólogo & 21 \\
\hline
\end{tabular}

...continuação

\begin{tabular}{ll}
\hline Palavra-chave & $\mathrm{n}$ \\
\hline representação social & 21 \\
adolescente & 20 \\
psicologia social & 20 \\
política pública & 17 \\
ensino superior & 15 \\
subjetividade & 15 \\
família & 14 \\
gênero & 13 \\
psicoterapia & 12 \\
direitos humanos & 11 \\
estudante universitário & 11 \\
psicologia escolar & 11 \\
psicologia histórico-cultural & 11 \\
sexualidade & 11 \\
deficiência & 10 \\
história da psicologia - Brasil & 10 \\
saúde pública & 10 \\
\hline
\end{tabular}

Tabela 7.

Palavras-chave mais frequentes.

\begin{tabular}{lc}
\hline palavra-chave & $\mathrm{n}$ \\
\hline psicologia & 102 \\
psicanálise & 71 \\
saúde mental & 67 \\
avaliação psicológica & 43 \\
trabalho & 42 \\
subjetividade & 40 \\
representação social & 36 \\
adolescente & 34 \\
psicologia social & 33 \\
atuação do psicólogo & 32 \\
formação do psicólogo & 31 \\
história da psicologia & 25 \\
política pública & 24 \\
família & 24 \\
adolescência & 24 \\
reforma psiquiátrica & 23 \\
gênero & 21 \\
psicoterapia & 20 \\
criança & 18 \\
psicólogo & 17 \\
\hline
\end{tabular}


Psicologia: Ciência e Profissão 2020 v. 40, e237692, 1-17.

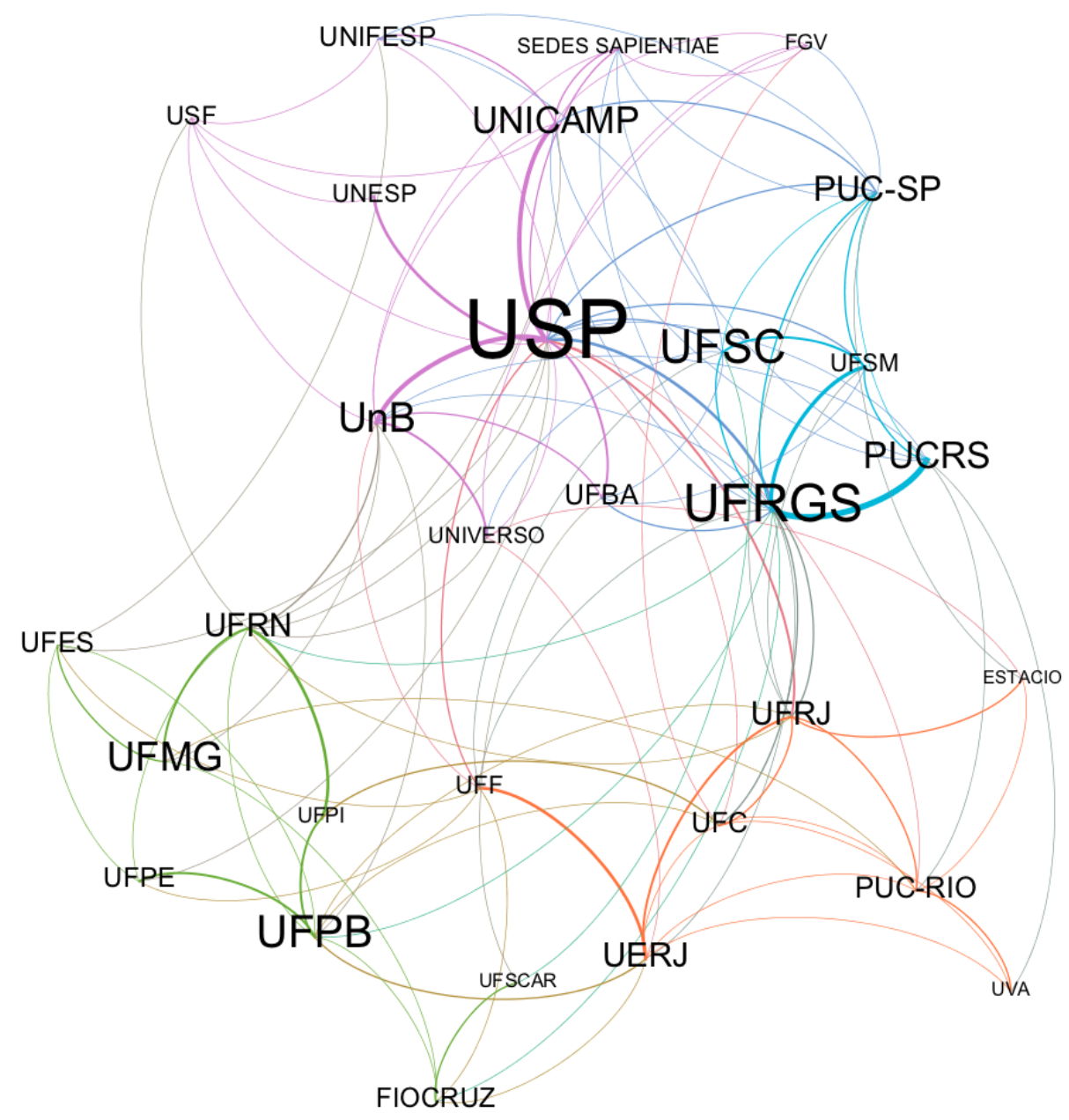

Figura 1.

Rede de universidades. 


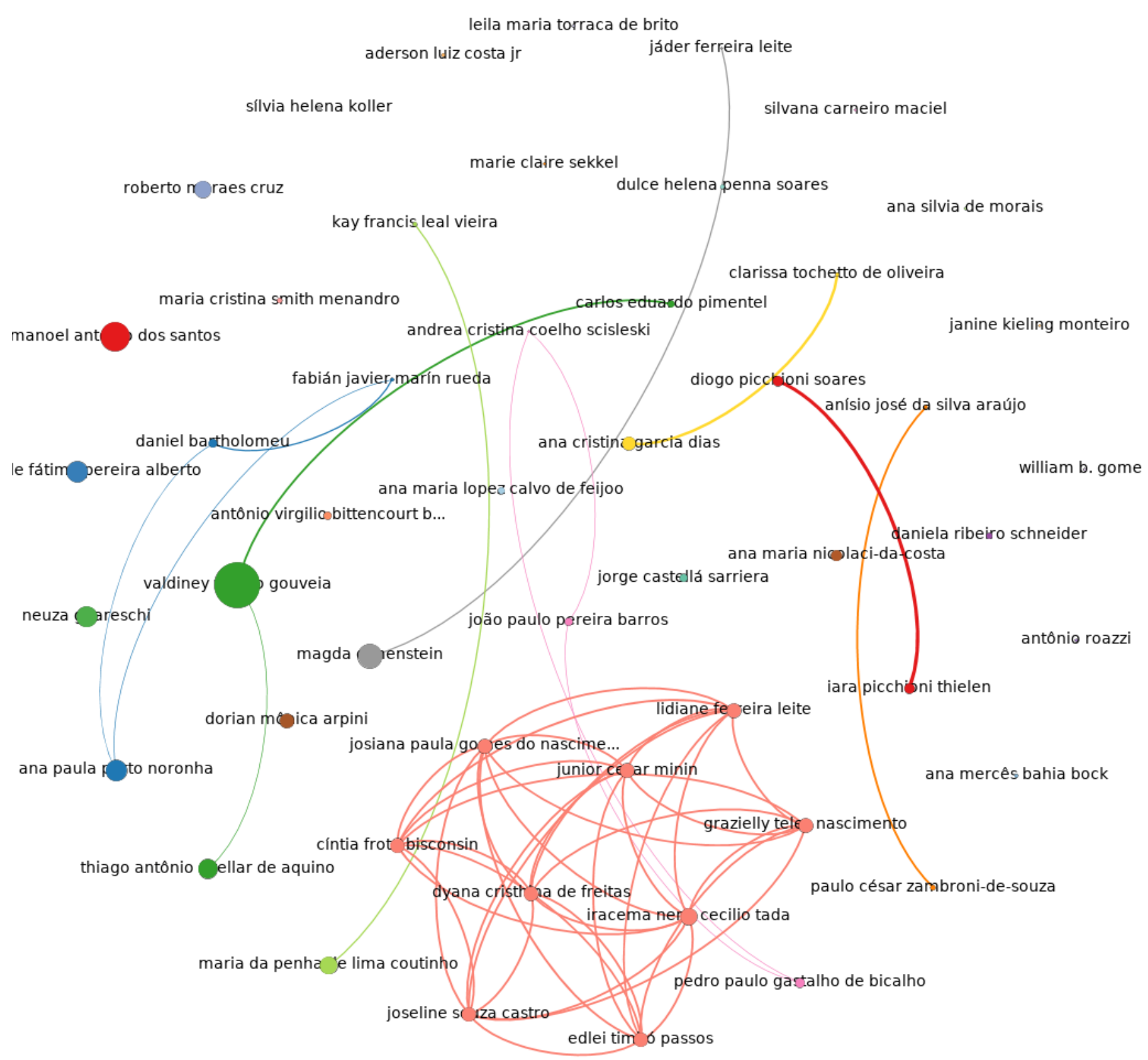

Figura 2.

Rede de autores. 


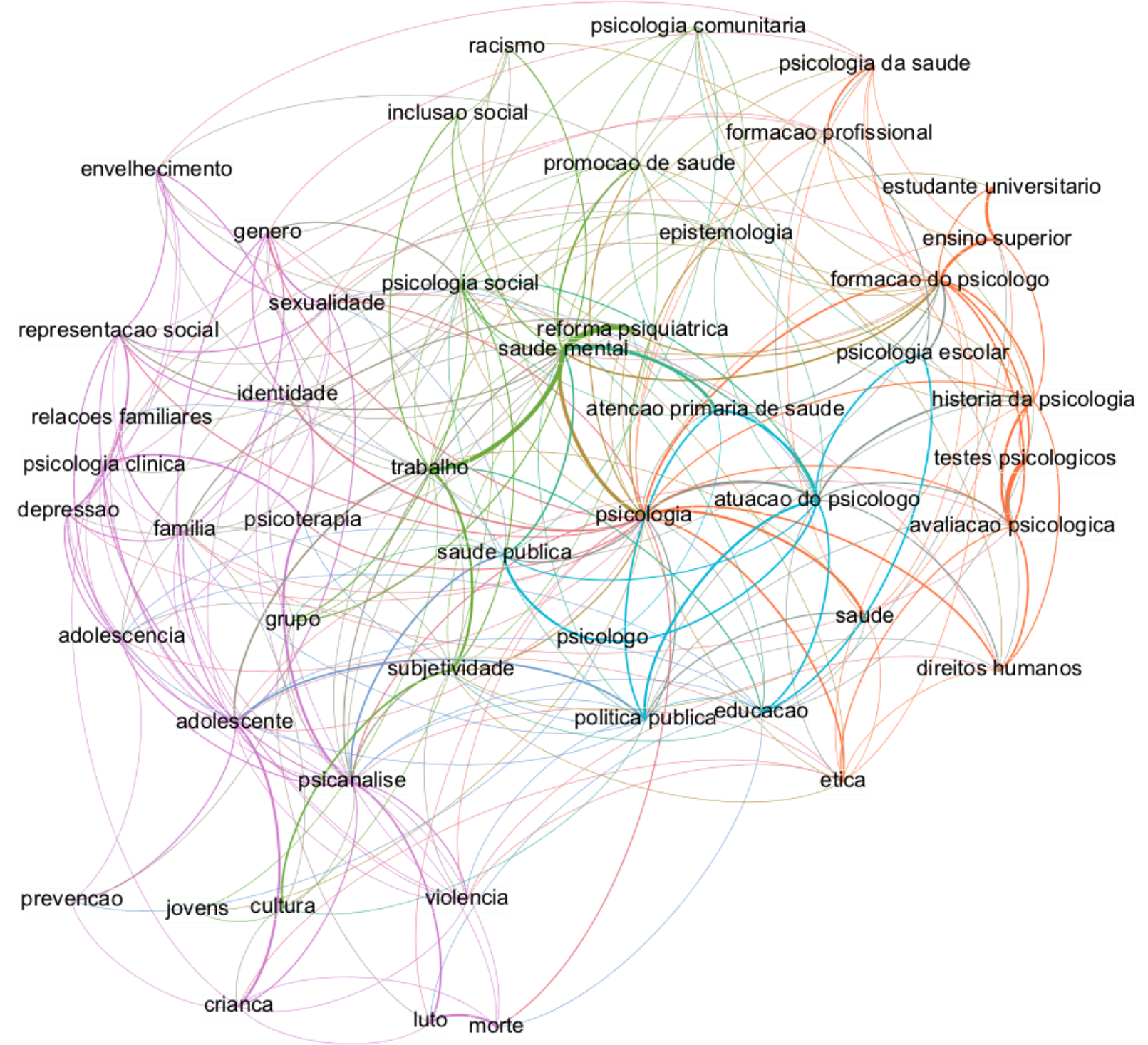

Figura 3.

Rede de palavras-chave.

\section{Discussão}

Os 40 anos da PCP mostram o desenvolvimento da área da Psicologia no Brasil e as características que a divulgação científica foi tomando ao longo desse período. Nos primeiros anos, enquanto o meio de envio dos manuscritos era os correios e a publicação era somente impressa, o número de artigos publicados era substancialmente menor (conforme observado na Tabela 1). Posteriormente, com a possibilidade de envio por correio eletrônico ou plataformas, como a SciELO, notabiliza-se o crescente número de autores e artigos. Quando se comparam os dois primeiros períodos com os dois últimos, de 1984 a 2001 com 2002 a 2019, é possível avaliar que o número de artigos foi quatro vezes maior, e o de autores, seis vezes superior.

Embora não se possa considerar o fenômeno das tecnologias de informação e comunicação como único elemento para o aumento das publicações, deve ser destacada a sua importância. A organização da Capes em torno de um modelo de revista científica e a abertura de novos cursos de Psicologia (Gomes, 2010), seja como graduação ou pós-graduação, provavelmente também tenham influência no aumento expressivo de instituições que publicaram na PCP. 
Os dados por período demonstram que, nos primeiros oito anos da PCP, existe um predomínio de publicações oriundas de instituições localizadas na região Sudeste do país (Tabela 2). A presença de universidades do estado de São Paulo marca os anos iniciais da revista, e, ainda que se observe a crescente influência de instituições de outras regiões, a instituição com maior número de publicações é a Universidade de São Paulo (USP). Por sua vez, os períodos subsequentes revelam o aumento de artigos provenientes de universidades da região Sul, com ênfase na Universidade Federal do Rio Grande do Sul (UFRGS) e na Universidade Federal de Santa Catarina (UFSC).

O total dos artigos produzidos por instituição evidencia a diferença entre as regiões do país, sendo que, das 26 universidades que registram maior número de publicações, 20 são do Sudeste (13) ou Sul (7). O restante das publicações se concentra em instituições da região Nordeste (5) e Centro-Oeste (1). A baixa presença de pesquisas realizadas nas regiões Nordeste e Centro-Oeste, assim como o fato de nenhuma universidade da região Norte estar presente entre as 26 com mais publicações, expõe algumas das características da pesquisa no país que também foram encontrados em investigações bibliométricas de outras áreas do conhecimento (Botelho, Brasil, \& Hoelz, 2019) e de subdivisões da própria Psicologia (Souza-Filho, Belo, \& Gouveia, 2006).

Entre os possíveis motivos dessa disparidade está o financiamento, que segue orientado para os grandes centros urbanos e com maior renda per capita do país. O caráter histórico da Psicologia de centrar-se em estudos no ambiente urbano (Calegare, 2015) também pode ser considerado no momento de entender a dinâmica das publicações.

Quando se comparam as instituições que publicam na PCP, evidencia-se também a diferença entre as universidades públicas, sejam estaduais (3) ou federais (16), e as privadas (6). A concentração da pesquisa em universidades públicas e, logo, o investimento que estas recebem servem como parâmetros para entender a importante diferença entre autores de instituições privadas e públicas no momento de publicar. Em contrapartida, é possível notar que o número de instituições privadas vem aumentando suas publicações nos últimos anos, porém o reflexo nos números totais nesta revista ainda é incipiente.

A localização dos cursos stricto sensu pelo país pode ser um dos motivos para o predomínio de artigos publicados oriundos de instituições do Sul, Sudeste e Centro-Oeste. Conforme os dados fornecidos pela Capes, existem 42 programas stricto sensu na região Sudeste, enquanto o restante das regiões contabiliza um total de 62 programas (Capes, 2019). Além do fator quantitativo, verifica-se que os programas de pós-graduação com notas maiores estão concentrados de forma assimétrica, sendo que entre os nove programas mais bem qualificados (extratos 7 e 6) apenas três se encontram fora do eixo Sul-Sudeste (Capes, 2017).

Os autores mais prolíficos durante os quatro períodos observados, assim como no resultado total de publicações/autor, estavam vinculados a instituições públicas. Em alguns casos, com base em consulta na plataforma Lattes, evidencia-se que houve trânsito entre o serviço privado, início da carreira, para posterior ingresso em universidades públicas. Ainda que esteja clara a expansão do setor privado no que concerne a pós-graduação, verifica-se que os programas são em sua maioria públicos, correspondendo a $72 \%$, enquanto as privadas respondem por $28 \%$ (Capes, 2017). A repercussão dessa preponderância de autores oriundos de universidades públicas ratifica algo amplamente reconhecido, que é a importância do Estado para a pesquisa nacional.

No que se refere às palavras-chave se, percebe-se tanto nos períodos individuais como no resultado total que existe o predomínio de termos genéricos relacionados ao campo da Psicologia. A utilização de termos relacionados a formação e atuação de psicólogos corrobora a preocupação da revista em contribuir com os aspectos científicos e profissionais que subsidiam a profissão, conforme apontado por diversos autores ao longo dos anos (Campos \& Bernardes, 2005; Gomes, 2010; Guedes, 2010). Neste mesmo sentido, a presença de palavras-chave que compõem a diversidade de linhas teóricas e campos de atuação profissional demonstra a representação plural dessas duas guias, ou seja, a ciência e o trabalho.

Dirigindo a análise para o desenvolvimento histórico, é possível notar que alguns termos são contínuos, enquanto outros apresentam sazonalidade bastante definida. Entre os exemplos que podem ser observados na Tabela 6 está a palavras-chave reforma psiquiátrica, a qual durante os anos de 2002-2010 teve relevância nas publicações, mas que no período seguinte deixa de estar entre as mais utilizadas. 
Esta sazonalidade sugere a importância dessa temática relacionada a saúde pública, compreendida não somente como campo de atuação profissional dos psicólogos como também frente de debate ético-político relacionado a políticas públicas de saúde mental. No período seguinte, anos de 2011-2019, surge pela primeira vez entre as palavras mais utilizadas o termo direitos humanos, que denota a transição para este tema que se impõe no debate público em geral e no campo específico da Psicologia.

A Tabela 7 demonstra que, além de termos gerais (psicologia, psicólogo, psicoterapia, atuação do psicólogo, formação do psicólogo), existem alguns que se encaixam emáreas temáticas de pesquisa (psicanálise, subjetividade, psicologia social, avaliação psicológica, representação social, gênero) ou populações específicas (adolescente, adolescência, família, criança). A transição para o modelo científico, com descritores mais específicos, pode levar, nos próximos anos, à diminuição de determinadas palavras-chave, como os dois termos, adolescente e adolescência, que poderiam ser alocados no mesmo ramo de significados.

O conjunto de palavras-chaves visualizadas nas Tabelas 6 e 7 corrobora o postulado de que a revista se mantém fiel à tradição institucional e científica, isto é, dá crescente espaço para a publicação de artigos cientificamente considerados de alto padrão para os termos nacional e, ao mesmo tempo, abre espaço para temáticas histórica e socialmente relevantes para a comunidade de psicólogos e brasileira.

Os dados que geraram as duas representações gráficas de colaboração auxiliam na compreensão de como instituições e autores se relacionam quando se tem como objeto de análise as publicações na PCP. Em relação às 30 instituições com maior número de colaborações, destaca-se a importância da regionalidade em suas interações, como no caso das universidades da região Nordeste, que se concentram em grande parte num mesmo grupo (linha verde da Figura 1), ou das universidades da região Sul (linha azul da Figura 1). Entre os possíveis fatores que podem afetar essa rede de colaboração está a interação de professores de acordo com a proximidade, seja para participação em bancas de defesa ou projetos que contemplem campos de pesquisa compartilhados. Outro elemento que surge como hipótese para entender essa interação são as relações de tutor-aluno e as redes que se estabelecem a partir destas, ou seja, com o seguimento de mestres e doutores para outras instituições e a publicação conjunta com os antigos tutores realizada a posteriori.

A rede de autores (Figura 2) demonstra que diversos autores reconhecidos no cenário nacional fazem parte do grupo que tem diversas publicações, entretanto não mantêm uma rede de coautoria constante na revista. Em outras palavras, o isolamento de autores prolíficos sugere que estes têm impacto na rede de autorias, ainda que não se consiga destacar outros autores que publiquem frequentemente com eles na PCP. Outro fator relevante diz respeito à quantidade de autores, pois, ao contrário de áreas em que existe número maior de autores e publicações, caso dos estudos epidemiológicos e outros da área da saúde, no campo das publicações da Psicologia brasileira não ocorre tal fenômeno. Os demais campos da saúde que realizam estudos multicêntricos em geral resultam em produções com mais autores, o que pode ser um dos fatores para explicar uma rede com menos colaboradores.

A Figura 3 apresenta a rede de relações entre as palavras-chave publicadas na PCP e demonstra quatro grandes núcleos. Entre estes, encontra-se a formação de cor laranja, que congrega algumas das subáreas da Psicologia, como avaliação psicológica, história da disciplina e Psicologia da Saúde, e temas basilares da formação, como direitos humanos, ética e formação do psicólogo. As linhas de cor azul claro formam o núcleo que tem o nó "atuação do psicólogo" como ponto central e que se associa a algumas das possibilidades para o profissional no âmbito da educação (Psicologia Escolar, educação) e da saúde pública (política pública, saúde pública, atenção primaria de saúde).

Por sua vez, os nós de cor verde se vinculam à Psicologia Social e Comunitária, assim como aos principais temas socialmente relevantes em que essa subárea costuma se deter: racismo, inclusão social, reforma psiquiátrica, trabalho e subjetividade. O núcleo roxo é o que apresenta maior número de palavras-chave, vinculando as palavras psicanálise, psicologia clínica e psicoterapia a outras que se associam a população/ fases do desenvolvimento (crianças, adolescente, adolescência, jovens, família, envelhecimento) e temas centrais para o campo da psicologia (depressão, luto, morte, sexualidade, gênero e violência).

Os diferentes agrupamentos de palavras-chave demonstram temas que se associam construindo relações. 


\section{Considerações finais}

O levantamento bibliométrico realizado neste estudo apresenta dados importantes para aqueles que se interessam pela construção da Psicologia no Brasil. A PCP, devido a seu vínculo com o órgão de classe, em uma análise histórica que busque entender o desenvolvimento da Psicologia brasileira, é imprescindível. Desta maneira, ilustrar aqueles que foram os principais autores, instituições e palavras-chave que marcaram presença nestes 40 anos da revista é sublinhar os caminhos que foram seguidos até a conformação atual.

No conjunto de dados recolhidos, sobretudo quando analisadas as palavras-chave, surge a pluralidade de temas como elemento que percorre os períodos da revista. Essa diversidade, que em outros contextos de edição talvez não seja o mais desejado, tendo em vista a segmentação do conhecimento, tem significado e relevância quando se compreende a complexidade de representar uma classe tão plural. Conforme citado anteriormente, a apresentação de artigos com enfoque científico e de atuação profissional está respaldada nos dados encontrados e assinala o comprometimento com o espectro de interesse daqueles que pertencem a esta categoria profissional. A escolha de publicar artigos mantendo-se a diversidade e tomando referenciais teóricos não associados ao pensamento científico neopositivista reforça uma segunda vez o olhar da Psicologia como disciplina signatária de uma epistemologia pluralizada (Abib, 2009).

\section{Referências}

Abib, J. A. D. (2009). Epistemologia pluralizada e história da Psicologia. Scientice Studia, 7(2), 195-208.

Antunes, M. A. M. (2006). A consolidação da psicologia no Brasil (1930-1962): sistematização de dados e algumas aproximações analíticas. Psicologia da Educação, 22, 79-94. http://pepsic.bvsalud.org/pdf/psie/n22/v22a05.pdf

Aria, M., \& Cuccurullo, C. (2017). Bibliometrix: An R-tool for comprehensive science mapping analysis. Journal of Informetrics, 11(4), 959-975. https://doi.org/10.1016/j.joi.2017.08.007

Bastian, M., Heymann, S., \& Jacomy, M. (2009). Gephi: An open source software for exploring and manipulating networks. http://www.aaai.org/ocs/index.php/ICWSM/09/paper/view/154

Baptista, M. T. D. (2010). A regulamentação da profissão psicologia: documentos que explicam o processo histórico. Psicologia: Ciência e Profissão, 30 (n. esp.), 170-191. https:// dx.doi.org/10.1590/S1414-98932010000500008

Botelho, A., Brasil, J. A., \& Hoelz, M. (2019). Tão longe, tão perto: Sociologia \& Antropologia no limiar de uma década. Sociologia \& Antropologia, 9(3), 717-739.https://www.scielo.br/scielo.php?pid=S2238$38752019000300717 \&$ script=sci_abstract\&tlng=pt

Calegare, M. G. A. (2015). Rural-urbano, estudos rurais e ruralidades: saberes necessários à psicologia social. In A. F. Lima, D. C. Antunes \& M. G. A. Calegare (Orgs.), A psicologia social e os atuais desafios ético-políticos no Brasil (pp. 473-457). Porto Alegre: Abrapso.

Callon, M., Law, J., \& Rip, A. (1986). Qualitative scientometrics. In M. Callon, J. Law \& A. Rip (Eds.), Mapping the dynamics of science and technology (pp. 103-123). Londres: Palgrave Macmillan UK.

Campos, R. H. F., \& Bernardes, L. H. G. (2005). A revista Psicologia: Ciência e Profissão: um registro da história recente da Psicologia brasileira. Psicologia: Ciência e Profissão, 25(4), 508-525. https://www.scielo.br/pdf/pcp/ v25n4/v25n4a02.pdf

Capes. (2017). Relatório de avaliação quadrienal 2013-2016. https://capes.gov.br/images/documentos/ Relatorios_quadrienal_2017/20122017-Psicologia_relatorio-de-avaliacao-2017_final.pdf

Capes. (2019). Documento de área. Área 37: Psicologia. https://www.gov.br/capes/pt-br/centrais-de-conteudo/ psicologia-pdf

de la Hoz-Correa, A., Muñoz-Leiva, F., \& Bakucz, M. (2018). Past themes and future trends in medical tourism research: a co-word analysis. Tourism Management, 65, 200-211. https://doi.org/10.1016/j.tourman.2017.10.001

De Langhe, R. (2017). Towards the discovery of scientific revolutions in scientometric data. Scientometrics, 110 (1), 505-519. http://doi.org/10.1007/s11192-016-2108-x

Farr, R. M. (2013). As raízes da psicologia social moderna. Petrópolis: Vozes. 
Feres-Carneiro, T. (2011). Departamento de Psicologia da Pontifícia Universidade Católica do Rio de Janeiro (PUCRio). In A. M. Jacó-Vilela (Org.), Dicionário histórico de instituições da psicologia no Brasil (pp. 149-150). Rio de Janeiro: Imago; Brasília: Conselho Federal de Psicologia.

Gomes, W. B., \& Gauer, G. (2010). Considerações sobre a história editorial recente da revista. Psicologia: Ciência e Profissão, 30 (n. esp.), 156-169. https://www.scielo.br/pdf/pcp/v30nspe/v30speca07.pdf

Guedes, M. C. (2010). De revista institucional a periódico científico: considerações para uma história de Psicologia: Ciência e Profissão. Psicologia: Ciência e Profissão, 30 (n. esp.), 148-155. https://www.scielo.br/ $\mathrm{pdf} / \mathrm{pcp} / \mathrm{v} 30 \mathrm{nspe} / \mathrm{v} 30$ speca06.pdf

Jacó-Vilela, A. M. (2012). História da psicologia no Brasil: uma narrativa por meio de seu ensino. Psicologia: Ciência e Profissão, 32 (n. esp.), 28-43. https://www.scielo.br/pdf/pcp/v32nspe/v32speca04.pdf

Lee, W. H. (2008). How to identify emerging research fields using scientometrics: an example in the field of information security. Scientometrics, 76 (3), 503-525. http:// doi.org/10.1007/s11192-007-1898-2

Meireles, F., Silva, D., \& Barbosa, R. (2019). Rscielo: a scraper for scientific journals hosted on Scielo. https://CRAN.R-project.org/package=rscielo

R Core Team. (2019). R: A language and environment for statistical computing. Viena: R Foundation for Statistical Computing. https://www.R-project.org/

Sampaio, M. I. C., Serradas, A., \& Santos, A. A. A. (2010). Psicologia: Ciência eProfissão: 30 anos registrando o avanço dapsicologiabrasileira. Psicologia:CiênciaeProfissão,30(n. esp),220-229.http://doi.org/10.1590/S1414-98932010000500011

Scarparo, H. (2011). Faculdade de Psicologia da Pontifícia Universidade Católica do Rio Grande do Sul (FAPSI/ PUCRS). In A. M. Jacó-Vilela (Org.), Dicionário histórico de instituições da psicologia no Brasil. (pp. 211-213). Rio de Janeiro: Imago; Brasília: Conselho Federal de Psicologia.

Soares, A. R. (2010). A psicologia no Brasil. Psicologia: Ciência e Profissão, 30 (n. esp), 8-41. https://doi.org/10.1590/ S1414-98932010000500002

Souza-Filho, M., Belo, R., Gouveia, V. V. (2006). Testes psicológicos: análise da produção científica brasileira no período 2000-2004. Psicologia: Ciência e Profissão, 26(3), 478-489

https://doi.org/10.1590/S1414-98932006000300011

Skinner, B.F. (1972). Tecnologia do ensino. São Paulo: E. P. U.

Uriona-Maldonado, M., dosSantos, R.N.M., \&Varvakis, G. (2012). State of the art on the systems ofinnovation research: a bibliometrics study up to 2009. Scientometrics, 91(3), 977-996. http://doi.org/10.1007/s11192-012-0653-5

Zanelli, J. C. (1995). Formação e atuação do psicólogo organizacional: uma revisão da literatura. Temas em Psicologia, 3(1), 95-107.

Zhou, P., \& Leydesdorff, L. (2006). The emergence of China as a leading nation in science. Research Policy, 35(1), 83-104. http://doi.org/10.1016/j.respol.2005.08.006

\section{Rodrigo de Oliveira Machado}

Doutor pelo Programa Pessoa e Sociedade no Mundo Contemporâneo - Psicologia Social, da Univesitat Autònoma de Barcelona, e pós-doutorando em Psicologia na Universidade Federal de Sergipe, Aracaju - SE. Brasil.

E-mail: rodrigomachado.psicologia@gmail.com

(1) https://orcid.org/0000-0001-8098-692X

\section{Jingyuan Yu}

Mestre em Comunicação Social pela Univesitat Pompeu Fabra (UPF) e doutorando pelo Programa de Doutorado em Pessoa e Sociedade no Mundo Contemporâneo da UAB, Barcelona. Espanha.

E-mail: jingyuan.yu@e-campus.uab.cat

(1) https://orcid.org/0000-0002-9400-4859 


\section{Miquel Domènech}

Doutor em Filosofia e Letras pela Univesitat Autònoma de Barcelona (UAB) e membro do Barcelona Science and Technology Studies group (STS-b) e Professor Titular de Psicologia Social no Departamento de Psicología Social da UAB. E-mail: miquel.domenech@uab.cat

(1) https://orcid.org/0000-0003-2854-3659

\section{Juan Muñoz-Justícia}

Doutor em Filosofia e Letras (Psicologia) pela Univesitat Autònoma de Barcelona (UAB) e professor associado no Programa de Pós-Graduação em Psicologia Social da UAB, Barcelona. Espanha.

E-mail: juan.munoz@uab.cat

(1) https://orcid.org/0000-0001-9985-4046

Endereço para envio de correspondência:

Universidade Federal de Sergipe. Av. Marechal Rondon, s/n, Departamento de Psicologia, Bloco Departamental IV. Jardim Rosa Elze. CEP: 49100-000. São Cristóvão - SE. Brasil.

Recebido 06/05/2020

Aceito 17/06/2020

Received 05/06/2020

Approved 06/17/2020

Recibido 06/05/2020

Aceptado 17/06/2020

Como citar: Machado, R. O., Yu, J., Domènech, M., \& Muñoz-Justícia, J. (2020). Análise Bibliométrica e de Rede dos 40 Anos da Revista Psicologia: Ciência e Profissão. Psicologia: Ciência e Profissão, 40, 1-17.

https://doi.org/10.1590/1982-3703003237692

How to cite: Machado, R. O., Yu, J., Domènech, M., \& Muñoz-Justícia, J. (2020). Bibliometric and Network Analysis of the 40 Years of the Journal Psicologia: Ciência e Profissão. Psicologia: Ciência e Profissão, 40, 1-17.

https://doi.org/10.1590/1982-3703003237692

Cómo citar: Machado, R. O., Yu, J., Domènech, M., \& Muñoz-Justícia, J. (2020). Análisis Bibliométrico y de Redes de los 40 Años de la Revista Psicologia: Ciência e Profissão. Psicologia: Ciência e Profissão, 40, 1-17.

https://doi.org/10.1590/1982-3703003237692 